\title{
Clinical Characteristics and Antimicrobial Susceptibility of Mycobacterium intracellulare and Mycobacterium abscessus Pulmonary Diseases: A Retrospective Study
}

\author{
Dongping Wang, ${ }^{1}$ Wenhong Lin $\mathbb{D}^{,}{ }^{2}$ Hongyan Cheng, ${ }^{1}$ Xundi Bao, ${ }^{1}$ Dongfang Xu, \\ Suo Liang, ${ }^{1}$ Yue Jiang, ${ }^{1}$ and Chao Wang ${ }^{1}$ \\ ${ }^{1}$ Department of Microbiology, Anhui Chest Hospital, Hefei, China \\ ${ }^{2}$ Department of Tuberculosis, Anhui Chest Hospital, Hefei, China
}

Correspondence should be addressed to Wenhong Lin; 627048134@qq.com

Received 15 July 2021; Revised 15 December 2021; Accepted 17 December 2021; Published 7 January 2022

Academic Editor: Maria De Francesco

Copyright (c) 2022 Dongping Wang et al. This is an open access article distributed under the Creative Commons Attribution License, which permits unrestricted use, distribution, and reproduction in any medium, provided the original work is properly cited.

\begin{abstract}
The incidence of nontuberculous mycobacteria (NTM) diseases is increasing every year. The present study was performed to investigate the clinical characteristics, CT findings, and drug susceptibility test (DST) results of patients diagnosed with M. intracellulare or M. abscessus nontuberculous mycobacterial pulmonary disease (NTMPD). This retrospective study included patients diagnosed with NTMPD due to M. intracellulare or M. abscessus for the first time at Anhui Chest Hospital between 01/ 2019 and 12/2021. The patients were grouped as M. intracellulare-NTMPD group or M. abscessus-NTMPD group. Clinical features, imaging data and DST data, were collected. Patients with $M$. intracellulare infection had a higher rate of acid-fast smears (66.1\% vs. $45.2 \%, P=0.032)$ and a higher rate of cavitation based on pulmonary imaging $(49.6 \%$ vs. $19.4 \%, P=0.002)$ than patients with $M$. abscessus infection, but both groups had negative TB-RNA and GeneXpert results, with no other characteristics significant differences. The results of DST showed that $M$. intracellulare had high susceptibility rate to moxifloxacin (95.9\%), amikacin (90.1\%), clarithromycin (91.7\%), and rifabutin (90.1\%). M. abscessus had the highest susceptibility rate to amikacin (71.0\%) and clarithromycin (71.0\%). The clinical features of $M$. intracellulare pneumopathy and $M$. abscessus pneumopathy are highly similar. It may be easily misdiagnosed, and therefore, early strain identification is necessary. $M$. intracellulare has a high susceptibility rate to moxifloxacin, amikacin, clarithromycin, and rifabutin, while M. abscessus has the highest susceptibility rate to amikacin and clarithromycin. This study provides an important clinical basis for improving the management of NTMPD.
\end{abstract}

\section{Introduction}

The incidence of nontuberculous mycobacteria (NTM) diseases is increasing every year, and it has become a public health concern due to the difficulty of diagnosis, the long course and high cost of treatment, and resistance to most antimicrobial drugs [1-4]. Over 90 species of NTM have been identified in humans [5]. NTM can be found in drinking water systems [6], domestic plumbing, showerheads, and potting soil [7-9]. The estimated NTM prevalence increased from 2.4 per 100,000 persons in 1980 to 15.2 per 100,000 persons in 2013 in the United States of America
[10]. Similar figures are observed in Canada [11], the United Kingdom [12], Denmark [13], and Germany [14]. The results of three consecutive epidemiological sampling surveys in China in 1990, 2000, and 2010 showed that the isolation rate of NTM was gradually increasing with $4.9 \%, 11.1 \%$, and $22.9 \%$, respectively [15]. NTM disease is clinically similar to tuberculosis and therefore can be misdiagnosed as such in the absence of microbiological identification [3]. Besides, most NTM are resistant to antimycobacterial drugs, resulting in poor efficacy of such drugs [16-20].

Among all types of NTM disease, nontuberculous mycobacterial pulmonary disease (NTMPD) is the most 
common one [21, 22]. In Western countries, the prevalence of NTMPD is higher than the prevalence of tuberculosis [23]. In the United States of America, Mycobacterium kasassii is the second most common cause of pulmonary infections, and those bacteria are also responsible for infections in the United Kingdom [2, 24]. Mycobacterium intracellulare is most isolated in China, with an isolation rate up to $40 \%-60 \%$ in Northern China $[25,26]$. In Southern China, Mycobacterium abscessus is also highly isolated in addition to Mycobacterium intracellulare [25, 26]. Still, a comprehensive analysis of the clinical characteristics, computed tomography (CT) findings, and drug susceptibility testing (DST) results of $M$. intracellulare and M. abscessus has been rarely reported in pulmonary disease.

Therefore, this study aimed to investigate the clinical characteristics, CT findings, and DST results of NTMPD patients diagnosed with $M$. intracellulare or $M$. abscessus infection at their first visit. The results of this study are expected to enrich the epidemiological data of NTM diseases and facilitate the early diagnosis and treatment of NTMPD.

\section{Materials and Methods}

2.1. Study Design and Patients. This retrospective study included patients with NTMPD who were first diagnosed with M. intracellulare infection or M. abscessus infection at Anhui Chest Hospital between January 2019 and December 2021. The study was approved by the ethics committee of Anhui Chest Hospital (approval number: K2021-007). Informed consent was obtained from the patients.

NTM strains were isolated from 772 inpatients during the three years; then, a total of 633 patients with M. intracellulare or M. abscessus isolated were initially included in the study for further research, and eventually 152 patients were analysed, of whom 121 had $M$. intracellulare pulmonary disease, and 31 had $M$. abscessus pulmonary disease.

All included patients had complete clinical data, appropriate laboratory findings, and imaging findings. Exclusion criteria were (1) NTM infection but no NTM disease, including tuberculosis [16], tumors, COPD, and chronic lung diseases such as bronchial and other diseases; (2) loss of follow-up after no definite diagnosis during hospitalization; (3) diagnosis of Mycobacterium tuberculosis lung disease complicated by NTM lung disease; and (4) diagnosis of NTM lung disease and readmission.

The diagnostic criteria of NTMPD are (1) samples not contaminated by exogenous factors; (2) there are respiratory and/or systemic symptoms; (3) there are spongy shadows, multifocal bronchiectasis, and multiple small nodular lesions on chest CT; and (4) two consecutive sputum $M y$ cobacterium cultures showing NTM of the same pathogen or one bronchial lavage fluid culture showing a positive result for NTM $(2+$ or $3+)$, or a lung tissue biopsy showing histopathological features of mycosis (positive granulomatous inflammation or positive acid-fast staining results) and positive NTM culture results, or biopsies showing histopathological features of the mycobacterial disease (positive granulomatous inflammation or positive acid-fast staining results) and positive NTM culture results in at least one of the sputum and bronchial lavage samples [16, 27].

2.2. NTM Culture and Species Identification. Sputum and bronchoalveolar lavage fluid samples were pretreated according to the Practice for Laboratory Testing of Tuberculosis [17] and cultured using the BACTEC MGIT 960 liquid culture method (Supplementary Table 1, and Figures 1 and 2). Strains with positive results by the colloidal gold method in the PNB identification medium (Zhuhai Baso Biotechnology Co., Ltd.) were initially identified using the MPB64 antigen detection kit (Hangzhou Genesis Biodetection \& Biocontrol Co., Ltd.). If the bacteria grew in the PNB medium or the MPB64 antigen test showed negative, the strain was tentatively identified as NTM (Figure 3). The strain preliminarily identified as NTM was then identified to species using the MeltPro Myco assay, which targets the intergenic transcribed spacer (ITS) region between the $16 \mathrm{~S}$ rRNA and $23 \mathrm{~S}$ rRNA genes of mycobacteria using a panmycobacterial primer set (Zeesan Biotech, Xiamen, China) [28] and the Bruker MALDI-TOF MS identifier [29, 30]. Six technicians with 6 to 15 years of experience, including two deputy chief technicians and four chief technicians, performed the NTM culture and species identification in this study.

2.3. DST of NTM. Broth microdilution method was performed according to Clinical and Laboratory Standards Institute (CLSI) guidelines [31, 32], using 96-well round bottom microtiter plates. Microplates and the microplate microbial susceptibility reader for mycobacteria (YK-909, Zhuhai Encode Medical Engineering Co., Ltd.) were used to test the antimicrobial susceptibility of the samples to 14 drugs, including amikacin $(1-64 \mu \mathrm{g} / \mathrm{mL})$, cefoxitin (4-160 $\mu \mathrm{g} / \mathrm{mL})$, clarithromycin $(0.5-64 \mu \mathrm{g} / \mathrm{mL})$, doxycycline $(0.5-128 \mu \mathrm{g} / \mathrm{mL})$, tobramycin $(0.5-64 \mu \mathrm{g} / \mathrm{mL})$, azithromycin $(1-32 \mu \mathrm{g} / \mathrm{mL})$, rifabutin $(0.5-32 \mu \mathrm{g} / \mathrm{mL})$, minocycline $(0.5-128 \mu \mathrm{g} / \mathrm{mL})$, linezolid $(0.5-32 \mu \mathrm{g} / \mathrm{mL})$, rifampicin $(1-16 \mu \mathrm{g} / \mathrm{mL})$, gatifloxacin $(0.06-8 \mu \mathrm{g} / \mathrm{mL})$, ethambutol $(2.5-20 \mu \mathrm{g} / \mathrm{mL})$, moxifloxacin $(0.125-16 \mu \mathrm{g} / \mathrm{mL})$, and compound sulfamethoxazole $(8-256 \mu \mathrm{g} / \mathrm{mL})$. The results were read according to the instructions on the microplate NTM DST kit (Zhuhai Encode Medical Engineering Co., Ltd.). The breakpoint of the minimum inhibitory concentration (MIC) of different drugs were read according to the rules listed in reference [31, 33-35], and CLSI M24-A2 [32] breakpoints were used for interpretation. The quality control strains were ATCC 927, ATCC 27853, and ATCC 29213. Three chief technicians with 10-12 years of experience performed the DST of NTM in the present study according to the previous study [32].

2.4. Data Collection. All data were collected from the electronic medical record system (Winning Health CIS 5.5.0.10). Patient demographic and clinical data were collected, including age, gender, underlying disease, comorbidities, family history, medication history, history of 


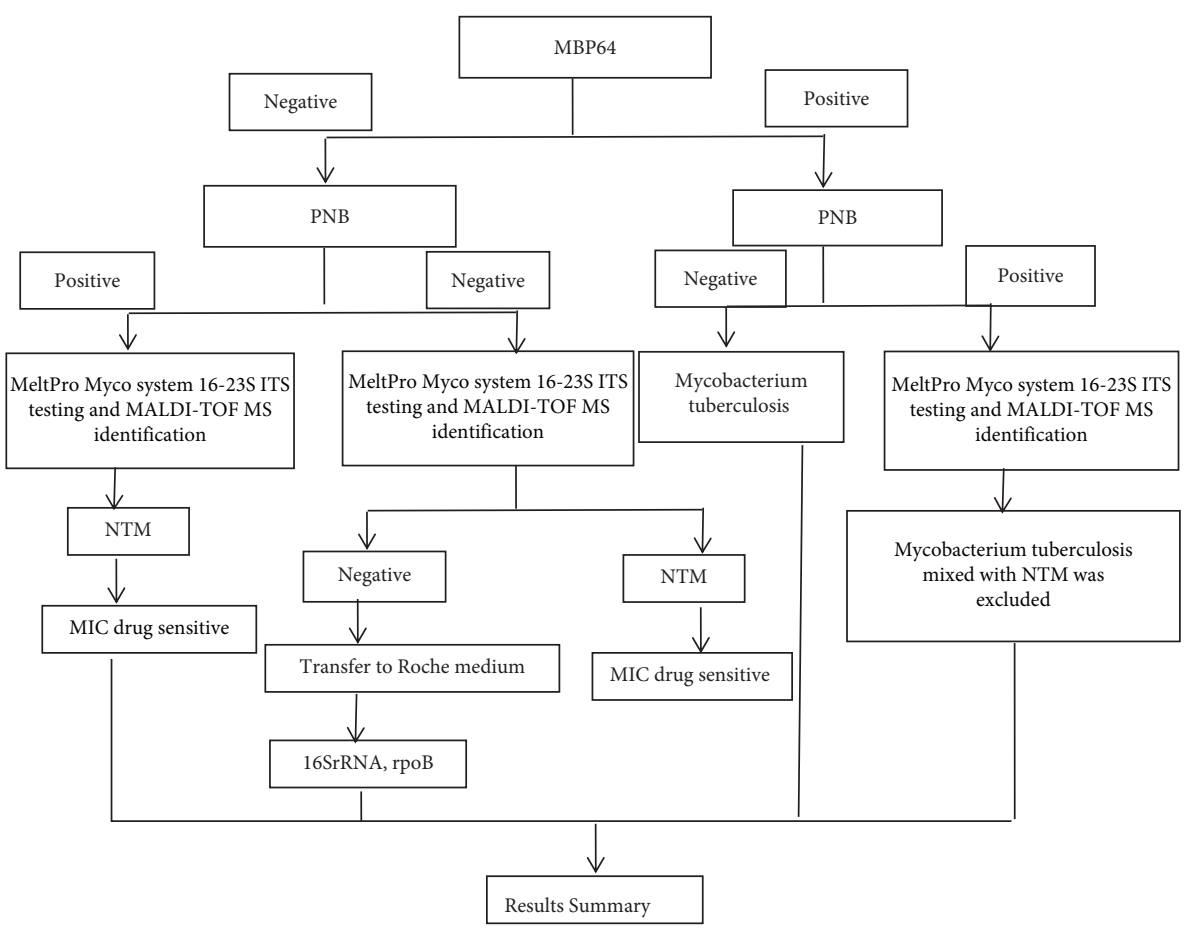

FIGURE 1: Culture and identification process of NTM.

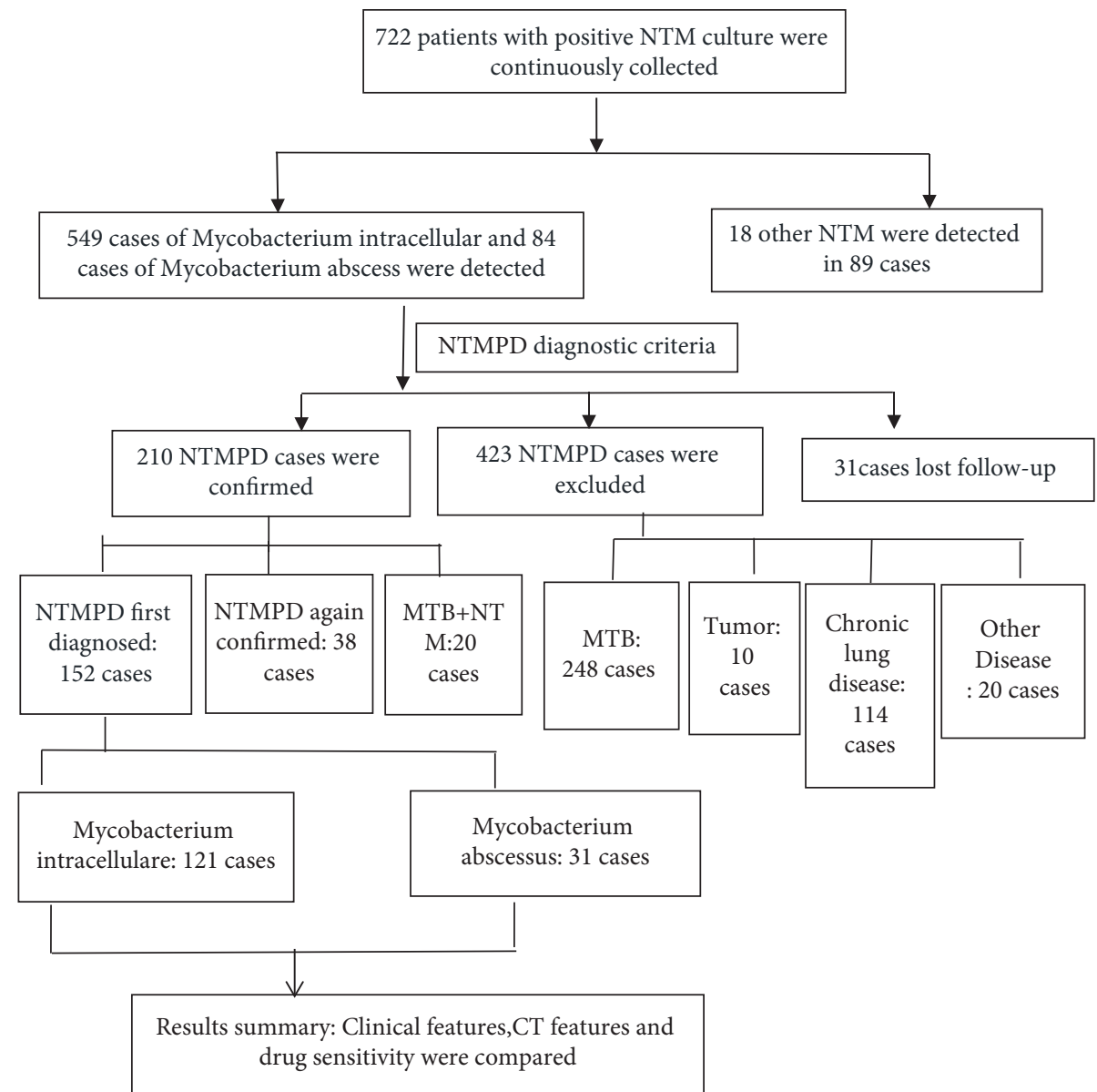

FIGURE 2: NTMPD sample inclusion. 


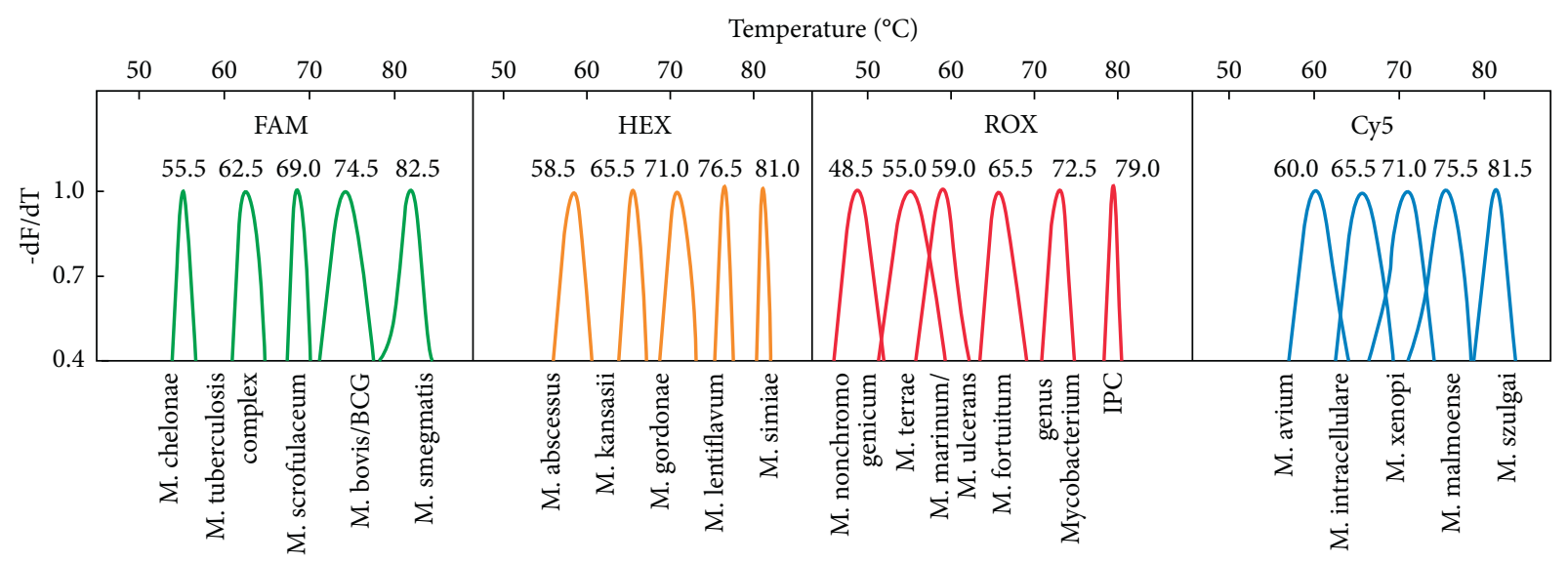

Figure 3: Melting peaks for each species in the MeltPro Myco assay in line with the 2D label strategy. To visually compare the melting temperature values of different Mycobacterium species, the melting curves showing the negative derivative of fluorescence intensity with respect to temperature were first normalized between 0 and 1, and then the data between 0.4 and 1 were plotted. MeltPro Myco assay: the MeltPro Myco assay targets the intergenic transcribed spacer (ITS) region between the 16S rRNA and 23S rRNA genes of mycobacteria using a panmycobacterial primer set. The MeltPro Myco assay includes 18 species-specific probes used to identify 17 NTM species and $M$. tuberculosis complex (MTBC), a genus-specific probe used to identify the Mycobacterium genus, and a set of primers and probes targeting the uninterrupted 229 bp sequence in the Mycobacterium bovis genome to distinguish Mycobacterium bovis and bacillus Calmette-Guérin (BCG) vaccine from M. tuberculosis, and a fragment of the Arabidopsis thaliana sucrose-proton symporter 2 (SUC2) gene serves as an internal positive control (IPC) [28]. In total, 21 labels were assigned to 19 mycobacterial species, the genus Mycobacterium, and IPC (Figure 3).

antituberculosis treatment, clinical symptoms, laboratory findings, imaging findings (lesion sites and morphological features), and DST results.

2.5. Statistical Analysis. SPSS 25.0 (IBM, Armonk, NY, USA) was used for statistical analysis. Continuous data that conformed to a normal distribution (according to the Shapiro-Wilk test) are expressed using means \pm standard deviations and were analysed using Student's $t$-test. Data that did not conform to a normal distribution were expressed as "medians (ranges)" and were analysed using the Mann-Whitney $U$-test. Categorical data were expressed as " $n$ (\%)" and were analysed using the chi-square test or Fisher's exact test. A two-sided $P$ value $<0.05$ was considered statistically significant.

\section{Results}

3.1. Clinical Characteristics of the Patients. A total of 633 patients with $M$. intracellulare or $M$. abscessus isolated were first identified, but 481 were excluded: 38 of them were diagnosed with NTMPD at the return visit, 20 with mixed Mycobacterium tuberculosis and NTM infection, 423 with NTM infection but no NTM disease, 248 of them were diagnosed with Mycobacterium tuberculosis infection, ten had neoplasms, 114 were diagnosed with chronic obstructive pulmonary disease, bronchiectasis, or other chronic pulmonary diseases, and 20 diagnosed with diseases other than NTMPD. In addition, 31 patients who were not diagnosed and lost to follow-up during hospitalization were excluded. Eventually, 152 patients were included, 121 of them had M. intracellulare pulmonary disease, and 31 had M. abscessus pulmonary disease.
Patients with $M$. intracellulare pulmonary disease had a higher rate of positive acid-fast smears than those with M. abscessus pulmonary disease $(66.1 \%$ vs. $45.2 \%, P=0.032)$. However, their TB-RNA and GeneXpert results were negative. A similar proportion (62.8\% vs. $64.5 \%)$ of patients with M. intracellulare and M. abscessus pulmonary disease received antituberculosis treatment for 1 to 36 months prior to diagnosis, with a mean median antituberculosis duration of 4 months and 6 months, respectively. There were no significant differences between the two groups in terms of age, sex, comorbidities, clinical symptoms, previous antituberculosis treatment, mean duration of antituberculosis treatment, and laboratory findings (all $P>0.05$ ) (Table 1).

3.2. Pulmonary Imaging Features. The comparison of the imaging findings between the two groups of patients is shown in Table 2. Patients with $M$. intracellulare lung disease had a higher rate of cavitation than patients with M. abscessus lung disease according to pulmonary imaging findings $(49.6 \%$ vs. $19.4 \%, P=0.002)$. There were no significant differences between the two groups in terms of lesion location, imaging findings of the lesions, mediastinal lymph node enlargement or calcification, emphysema, alveoli, lung injury, and bronchodilation (all $P>0.05$ ).

3.3. DST Results and Susceptibility Rates. The strains of M. intracellulare showed high susceptibility rate to moxifloxacin $(95.9 \%)$, amikacin $(90.1 \%)$, clarithromycin $(91.7 \%)$, and rifabutin (90.1\%). The strains of $M$. abscessus showed the highest susceptibility to amikacin (71.0\%) and clarithromycin (71.0\%). Table 3 shows the MIC range, MIC 50, and MIC 90 in the two groups. 
TABLE 1: Characteristics of the patients with NTMPD.

\begin{tabular}{|c|c|c|c|}
\hline & $\begin{array}{l}\text { Mycobacterium intracellulare } \\
n=121\end{array}$ & $\begin{array}{c}\text { Mycobacterium abscessus } \\
n=31\end{array}$ & $P$ value \\
\hline Age, years, mean $\pm S D$ & $65.0 \pm 10.2$ & $64.7 \pm 11.6$ & 0.893 \\
\hline Sex $(\%)$ & & & 0.936 \\
\hline Male & $65.3(79 / 121)$ & $64.5(20 / 31)$ & \\
\hline Female & $34.7(42 / 121)$ & $35.5(11 / 31)$ & \\
\hline \multicolumn{4}{|l|}{ Complications (\%) } \\
\hline Chronic obstructive pulmonary disease & $17.4(21 / 121)$ & $9.7(3 / 31)$ & 0.296 \\
\hline Bronchiectasis & $37.2(45 / 121)$ & $51.6(16 / 31)$ & 0.397 \\
\hline Diabetes & $9.1(11 / 121)$ & $16.1(5 / 31)$ & 0.322 \\
\hline \multicolumn{4}{|l|}{ Medication history } \\
\hline Received antituberculosis treatment before (\%) & $62.8(76 / 121)$ & $64.5(20 / 31)$ & 0.861 \\
\hline Mean median antituberculosis duration (months) & 4 & 5 & 0.974 \\
\hline Glucocorticoid (\%) & $16.5(20 / 121)$ & $19.4(6 / 31)$ & 0.709 \\
\hline \multicolumn{4}{|l|}{ Symptoms (\%) } \\
\hline Cough & $71.1(86 / 121)$ & $77.4(24 / 31)$ & 0.481 \\
\hline Expectoration & $61.2(74 / 121)$ & $61.3(19 / 31)$ & 0.989 \\
\hline Fever & $10.7(13 / 121)$ & $12.9(4 / 31)$ & 0.752 \\
\hline Hemoptysis & $15.7(19 / 121)$ & $19.4(6 / 31)$ & 0.625 \\
\hline \multicolumn{4}{|l|}{ Positive laboratory examination (\%) } \\
\hline T-SPOT & $23.4(11 / 47)$ & $20(2 / 10)$ & 0.713 \\
\hline Acid-fast smear & $66.1(80 / 121)$ & $45.2(14 / 31)$ & $0.032^{*}$ \\
\hline Tuberculosis DNA & $2.0(2 / 102)$ & $6.7(1 / 15)$ & 0.34 \\
\hline TB-RNA & $0(0 / 112)$ & $0(0 / 24)$ & - \\
\hline Xpert MTB/RIF & $0(0 / 83)$ & $0(0 / 15)$ & - \\
\hline Tuberculosis antibodies & $55.3(52 / 94)$ & $50(11 / 22)$ & 0.652 \\
\hline Hemoglobin, g/L, mean \pm SD & $112.35 \pm 27.30$ & $111.00 \pm 33.25$ & 0.816 \\
\hline Albumin, $\mathrm{g} / \mathrm{L}$, mean $\pm \mathrm{SD}$ & $34.81 \pm 4.51$ & $34.47 \pm 5.39$ & 0.716 \\
\hline Red blood cells, $\times 10^{12} / \mathrm{L}$, mean $\pm \mathrm{SD}$ & $4.02 \pm 0.66$ & $4.05 \pm 0.72$ & 0.824 \\
\hline White blood cells, $\times 10^{9} / \mathrm{L}$, mean \pm SD & $5.73 \pm 2.09$ & $5.96 \pm 2.40$ & 0.591 \\
\hline $\mathrm{ESR}, \mathrm{mm} / \mathrm{h}$, mean $\pm \mathrm{SD}$ & $32.87 \pm 26.11$ & $33.64 \pm 29.32$ & 0.852 \\
\hline
\end{tabular}

SD, standard deviation; ESR: erythrocyte sedimentation rate. $P<0.05$ were deemed statistically significant.

TABle 2: Pulmonary imaging findings of patients with NTMPD.

\begin{tabular}{|c|c|c|c|}
\hline Features, $n(\%)$ & Mycobacterium intracellulare $n=58$ & Mycobacterium abscessus $n=16$ & $P$ value \\
\hline \multicolumn{4}{|l|}{ Site of lesion } \\
\hline Left lung & $3(2.5)$ & 0 & $>0.999$ \\
\hline Right lung & $7(5.8)$ & 0 & 0.346 \\
\hline Both lungs & $111(91.7)$ & $31(100)$ & 0.215 \\
\hline Cord-like shadow & $39(32.2)$ & $9(29.0)$ & 0.732 \\
\hline Plaque-like shadow & $42(34.7)$ & $14(45.2)$ & 0.282 \\
\hline Nodular shadow & $45(37.2)$ & $10(32.3)$ & 0.61 \\
\hline Pleural thickening or pleural effusion & $28(23.1)$ & $9(29.0)$ & 0.495 \\
\hline Mediastinal lymph node enlargement or calcification & $21(17.4)$ & $3(9.7)$ & 0.411 \\
\hline Emphysema, bullae & $23(19.0)$ & $6(19.4)$ & 0.965 \\
\hline Lung damage & $7(5.8)$ & $1(3.2)$ & $>0.999$ \\
\hline Bronchiectasis & $45(37.2)$ & $16(51.6)$ & 0.144 \\
\hline Cavity & $60(49.6)$ & $6(19.4)$ & $0.002^{*}$ \\
\hline
\end{tabular}

The data are shown as $n$ (\%). $P<0.05$ were deemed statistically significant.

\section{Discussion}

The incidence of NTM disease is increasing year by year, but analysis of the clinical features, CT findings, and DST results of Chinese patients has rarely been reported. The results of this study indicate that the clinical features of $M$. intracellulare pneumopathy and M. abscessus pneumopathy are highly similar, necessitating the identification of molecular biology strains. DST results showed that M. intracellulare was highly sensitive to moxifloxacin, amikacin, clarithromycin, and rifabutin, while $M$. abscessus had the highest sensitivity to amikacin and clarithromycin.

Studies indicated that it takes a long time to diagnose NTMPD (7 to 8 years on average), and most patients were first misdiagnosed with pulmonary tuberculosis $(92.8 \%)$ $[36,37]$. A study in Iran showed that among 714 positive 
TABLE 3: Comparison of DST results and susceptibility rates to 14 drugs in NTMPD.

\begin{tabular}{|c|c|c|c|c|c|c|}
\hline \multirow{2}{*}{ Drug name, $n(\%)$} & \multicolumn{3}{|c|}{$\operatorname{MIC}(\mu \mathrm{g} / \mathrm{mL})$} & \multicolumn{3}{|c|}{ Number (\%) } \\
\hline & MIC range & MIC 50 & MIC 90 & $S$ & $I$ & $R$ \\
\hline \multicolumn{7}{|c|}{ Mycobacterium intracellulare, $n=121$} \\
\hline Amikacin & $<1-64$ & 1 & 16 & $109(90.1)$ & $5(4.1)$ & $7(5.8)$ \\
\hline Azithromycin & $1-32$ & 4 & 32 & $9(7.4)$ & $90(74.4)$ & $22(18.2)$ \\
\hline Clarithromycin & $<0.5-64$ & 0.5 & 64 & $111(91.7)$ & $2(1.7)$ & $8(6.6)$ \\
\hline Linezolid & $<0.5-32$ & 8 & 32 & $34(28.1)$ & $68(56.2)$ & $19(15.7)$ \\
\hline Moxifloxacin & $0.125-16$ & 0.5 & 2 & $116(95.9)$ & $3(2.5)$ & $2(1.7)$ \\
\hline Rifabutin & $<0.5-32$ & 0.5 & 16 & $109(90.1)$ & 0 & $12(9.9)$ \\
\hline Rifampicin & $<1-16$ & 1 & 16 & $100(82.6)$ & $8(6.6)$ & $13(10.7)$ \\
\hline Gatifloxacin & $0.06-8$ & 1 & 4 & - & - & - \\
\hline Doxycycline & $4->128$ & 32 & $>128$ & - & - & - \\
\hline Minocycline & $<4->128$ & 32 & 64 & - & - & - \\
\hline Sulfamethoxazole & $16->256$ & 192 & $>256$ & - & - & - \\
\hline Cefoxitin & $4-160$ & 32 & $>160$ & - & - & - \\
\hline Tobramycin & $<0.5-64$ & 4 & 32 & - & - & - \\
\hline Ethambutol & $<2.5->20$ & 2.5 & 20 & $61(50.4)$ & $33(27.3)$ & $27(22.3)$ \\
\hline \multicolumn{7}{|c|}{ Mycobacterium abscessus, $n=31$} \\
\hline Amikacin & $<1-64$ & 4 & 64 & $22(71.0)$ & $4(12.9)$ & $5(16.1)$ \\
\hline Azithromycin & $<1-32$ & 16 & 32 & $10(32.3)$ & $6(19.4)$ & $15(48.4)$ \\
\hline Clarithromycin & $<0.5-64$ & 0.5 & 64 & $22(71.0)$ & $2(6.5)$ & $7(22.6)$ \\
\hline Linezolid & $2-32$ & 8 & 32 & $16(51.6)$ & $9(29.0)$ & $6(19.4)$ \\
\hline Moxifloxacin & $0.125-16$ & 4 & 16 & $9(29.0)$ & $7(22.6)$ & $15(48.4)$ \\
\hline Rifabutin & $<0.5-2$ & 2 & 32 & - & - & - \\
\hline Rifampicin & $<1-16$ & 16 & 16 & - & - & - \\
\hline Gatifloxacin & $0.06-8$ & 2 & 8 & - & - & - \\
\hline Doxycycline & $16->128$ & $>128$ & $>128$ & 0 & 0 & $31(100)$ \\
\hline Minocycline & $<4->128$ & 64 & $>128$ & - & - & - \\
\hline Sulfamethoxazole & $32->256$ & $>256$ & $>256$ & $1(3.2)$ & $1(3.2)$ & $29(93.5)$ \\
\hline Cefoxitin & $4-160$ & 48 & 96 & $21(67.7)$ & 0 & $10(32.3)$ \\
\hline Tobramycin & $<0.5-64$ & 32 & 64 & $3(9.7)$ & $5(16.1)$ & $23(74.2)$ \\
\hline Ethambutol & $<2.5->20$ & $>20$ & $>20$ & - & - & - \\
\hline
\end{tabular}

S, susceptible; I, intermediary; R, resistant. Because there is no recognized breakpoint, the drug resistance rate cannot be calculated.

acid-fast bacilli from TB-suspected cases, 95 isolates were identified as NTM (13.3\%) [38]. Furthermore, many NTMPD patients were infected by a wide range of atypical mycobacteria [39]. Therefore, it is of great importance to differentiate NTM from Mycobacterium tuberculosis and to make reliable identification in clinical practice to reduce misdiagnosis and improve treatment efficiency. Before diagnosis, most patients had received repeated antituberculous drug treatment. Our research indicated that most patients were misdiagnosed before they were diagnosed with NTMPD as nearly $70 \%$ of the patients in both groups had a history of antituberculosis treatment, which lasted for 4 to 5 months on an average and 3 years on the longest, which was consistent with the previous study. The high rate of misdiagnosis of NTMPD, the difficulty in confirming the diagnosis, and the high cost of treatment when patients are misdiagnosed make it important to find diagnostic markers for NTMPD.

In the present study, out of 633 patients infected with M. intracellulare or M. abscessus, only 152 were diagnosed with NTM disease at the first visit, 38 were diagnosed with NTMPD at the second visit, 20 were diagnosed with mixed infection with Mycobacterium tuberculosis and NTM, 31 were not diagnosed and lost to contact, and the remaining 423 were excluded due to the absence of NTMPD. Such results suggest that NTM strains isolated in the laboratory only prove the possibility of NTM infection. Clinicians should still identify NTMPD based on diagnostic criteria.

Patients of both groups had a high positive rate of acidfast smears. Those with $M$. intracellulare pulmonary disease having a positive rate of $66.1 \%$, and those with $M$. abscessus pulmonary disease showing $45.2 \%$, which is in agreement with the findings of Riello et al. [40]. However, TB-RNA and GeneXpert results were negative in both groups, suggesting that if a patient has a positive acid-fast smear and a negative TB-RNA and GeneXpert result, then he/she may have NTM disease.

At imaging, lesions of NTMPD are various and diffuse, and double lung lesions are more common in NTMPD than in pulmonary tuberculosis $[16,26,41,42]$. In this study, patients with $M$. abscessus pulmonary disease had a higher rate of cavitation than patients with $M$. intracellulare pulmonary disease based on lung imaging findings (49.6\% vs. $19.4 \%, P=0.002)$. There were no significant differences between the two groups in lesion location, lesion severity, the characteristics of pulmonary shadow, pleural thickening or pleural effusion, and bronchiectasis, which was in line with the results of a previous study [26].

Most NTM are resistant to traditional antituberculosis drugs, and their resistance patterns vary greatly with 
different strains. The therapeutic effect after infection mainly depends on the strains identified [43-45]. The consensus of experts on diagnosis and treatment of nontuberculous mycobacterium disease [16] clearly points out that drug sensitivity test before treatment is still very important, and the consensus believes that the correlation between drug sensitivity test results and clinical effect is still difficult to determine, but it is still suggested to base the drug sensitivity results and medication history as far as possible when making chemotherapy regimen for NTM disease. $M$. intracellulare belongs to slowly growing mycobacteria (SGM) of nontuberculous mycobacteria. Expert consensus [16] and An Official ATS/ERS/ESCMID/IDSA Clinical Practice Guideline [27] suggested that macrolides are -the only effective antibacterial agents for the treatment of Mycobacterium avium-intracellulare infection, and the recommended drugs include clarithromycin, azithromycin, amikacin, rifabutin, rifampicin, and ethambutol. The results of drug sensitivity test in this study showed that the drug sensitivity rate of Mycobacterium intracellulare was clarithromycin (91.7\%), amikacin (90.1\%), rifabutin $(90.1 \%)$, rifampicin (82.6\%), ethambutol (50.4\%), azithromycin (18.2\%), and moxifloxacin (95.9\%), respectively. The authors of $[31,46-48]$ reported the drug sensitive rate of clarithromycin (94.2-97.3\%), amikacin (57.7-99.4\%), rifabutin (95.3\%), rifampicin (34.3-75.4\%), ethambutol (8.7-75.1\%), azithromycin (85.5\%), and moxifloxacin (5.5-89.6\%). Except azithromycin, the drug sensitivity results in this study were in good agreement with literature reports. In this study, the sensitivity rate of azithromycin (18.2\%) was low, which was significantly different from that reported in the literature. The results for clarithromycin predict those for azithromycin, for which testing is problematic as a result of poor solubility at the high concentrations of drug that must be used [32]. The susceptibility rate of clinical Mycobacterium intracellulare isolates to moxifloxacin was $95.9 \%$. Wang et al. [46] reported that fluoroquinolone moxifloxacin also had good antibacterial activity against Mycobacterium intracellulare in vitro. This study was consistent with literature reports, but moxifloxacin was not among the consensus-recommended drugs.

M. abscessus belongs to rapidly growing mycobacteria (RGM) of nontuberculous mycobacteria. M. abscessus occupies the largest proportion of RGM that is naturally resistant to and possess acquired resistance to most commonly used antibiotics, commonly treated with antituberculous drugs; also, M. abscessus is known as a nightmare bacterium. Expert consensus [16] and An Official ATS/ERS/ESCMID/ IDSA Clinical Practice Guideline [27] suggested that the treatment of $M$. cheloniae abscess includes clarithromycin, azithromycin, amikacin, and cefoxitin. The drug sensitivity test results of this study showed that the drug sensitivity rate of M. abscessus was clarithromycin (71.0\%),azithromycin $(32.3 \%)$, amikacin $(71.0 \%)$, linezolid (51.6\%), and cefoxitin $(67.7 \%)$. There are great differences in laboratory drug susceptibility data around the world. The authors of [31, 49-52] reported the susceptibility rates of $M$. abscessus to clarithromycin (62-75.5\%), azithromycin (95\%), amicacin (15.4-100\%), cefoxitin (0-54\%), linezolid (14.7-98.1\%). In this study, the in vitro antibacterial activities of amikacin (71.0\%) and clarithromycin (71.0\%) were similar to those reported in [31]. Some natural drug resistance mechanisms of Mycobacterium abscessus include waxy and opaque cell walls, drug delivery system, antibiotic modification/inactivation enzyme, and genetic polymorphism of target genes [53]. Macrolides are basic drugs for the treatment of $M y$ cobacterium intracellulare and Mycobacterium abscessus, and clinical isolates can detect erM(41) (induced macrolide resistance) and/or $23 \mathrm{~S}$ rRNA point mutation (constitutive macrolide resistance) information, which can obtain the sensitivity of macrolides $[51,53]$.

This study has limitations. First, it studied only two NTM and had a small sample size so that the results might be biased. Second, due to limited testing competence and methodology, the study did not investigate the resistancerelated genetics.

\section{Conclusions}

In conclusion, there are still many difficulties in diagnosing and treating NTMPD, and NTM disease can be easily misdiagnosed. Elderly people are more likely to have NTMPD, and delayed diagnosis of NTMPD and improper antituberculosis treatment are common in clinical practice due to highly similar clinical symptoms and signs, laboratory examinations, and imaging. $M$. intracellulare has a high susceptibility rate to moxifloxacin, amikacin, clarithromycin, and rifabutin, while $M$. abscessus has the highest susceptibility rate to amikacin and clarithromycin.

\section{Data Availability}

All the data generated or analysed during this study are included in this published article.

\section{Conflicts of Interest}

The authors declare no conflicts of interest regarding the publication of this paper.

\section{Authors' Contributions}

Dongping Wang contributed to the design and drafted the manuscript. Wenhong Lin contributed to the conception and critically revised the manuscript. Hongyan Cheng contributed to acquisition of the data and analysis of the data. Xundi Bao contributed to analysis of the data. Dongfang $\mathrm{Xu}$ and Suo Liang contributed to interpretation of the data. Yue Jiang and Chao Wang contributed to acquisition of the data. All the authors have read and approved the final manuscript.

\section{Acknowledgments}

This study was supported by the Scientific Research Project of Anhui Provincial Health Commission (grant no. AHWJ2021b072) and Anhui Province Chest Hospital (Provincial Tuberculosis Prevention and Control Institute) Scientific Research Project (grant no. 2020kj009B). 


\section{Supplementary Materials}

Supplementary Table 1: sample composition of sputum and bronchoalveolar lavage fluid in the two groups. (Supplementary Materials)

\section{References}

[1] R. Reves and N. W. Schluger, "Update in tuberculosis and nontuberculous mycobacterial infections 2013," American Journal of Respiratory and Critical Care Medicine, vol. 189, no. 8, pp. 894-898, 2014.

[2] D. E. Griffith, T. Aksamit, B. A. Brown-Elliott et al., "An official ATS/IDSA statement: diagnosis, treatment, and prevention of nontuberculous mycobacterial diseases," American Journal of Respiratory and Critical Care Medicine, vol. 175, no. 4, pp. 367-416, 2007.

[3] C. N. Ratnatunga, V. P. Lutzky, A. Kupz et al., "The rise of non-tuberculosis mycobacterial lung disease," Frontiers in Immunology, vol. 11, p. 303, 2020.

[4] J. O. Falkinham, "3rd ecology of nontuberculous mycobacteria-where do human infections come from?" Seminars in Respiratory and Critical Care Medicine, vol. 34, no. 1, pp. 95-102, 2013.

[5] W. Hoefsloot, J. Van Ingen, C. Andrejak et al., "The geographic diversity of nontuberculous mycobacteria isolated from pulmonary samples: an NTM-NET collaborative study," European Respiratory Journal, vol. 42, no. 6, pp. 1604-1613, 2013.

[6] P. Mohajeri, L. Yazdani, A. H. Shahraki et al., "Verification of frequency in species of nontuberculous mycobacteria in kermanshah drinking water supplies using the PCR-sequencing method," Microbial Drug Resistance, vol. 23, no. 3, pp. 359-364, 2017.

[7] J. R. Honda, N. A. Hasan, R. M. Davidson et al., "Environmental nontuberculous mycobacteria in the Hawaiian islands," PLoS Neglected Tropical Diseases, vol. 10, no. 10, Article ID e0005068, 2016.

[8] J. R. Honda, R. Virdi, and E. D. Chan, "Global environmental nontuberculous mycobacteria and their contemporaneous man-made and natural niches," Frontiers in Microbiology, vol. 9, p. 2029, 2018.

[9] Y. Nishiuchi, T. Iwamoto, and F. Maruyama, "Infection sources of a common non-tuberculous mycobacterial pathogen, Mycobacterium avium complex," Frontiers of Medicine, vol. 4, p. 27, 2017.

[10] M. J. Donohue and L. Wymer, "Increasing prevalence rate of nontuberculous mycobacteria infections in five states, 2008-2013," Annals of the American Thoracic Society, vol. 13, no. 12, pp. 2143-2150, 2016.

[11] S. K. Brode, A. Marchand-Austin, F. B. Jamieson, and T. K. Marras, "Pulmonary versus nonpulmonary nontuberculous mycobacteria, Ontario, Canada," Emerging Infectious Diseases, vol. 23, no. 11, pp. 1898-1901, 2017.

[12] J. E. Moore, M. E. Kruijshaar, L. P. Ormerod, F. Drobniewski, and I. Abubakar, "Increasing reports of non-tuberculous mycobacteria in england, wales and northern Ireland, 1995-2006," BMC Public Health, vol. 10, no. 1, p. 612, 2010.

[13] C. Andréjak, V. Ø. Thomsen, I. S. Johansen et al., "Nontuberculous pulmonary mycobacteriosis in Denmark," American Journal of Respiratory and Critical Care Medicine, vol. 181, no. 5, pp. 514-521, 2010.
[14] R. Diel, J. Jacob, N. Lampenius et al., "Burden of non-tuberculous mycobacterial pulmonary disease in Germany," European Respiratory Journal, vol. 49, no. 4, 2017.

[15] L. X. Wang, S. M. Cheng, and M. T. Chen, "[Report on fifth national tuberculosis epidemiological sampling survey in 2010]," Chinese Journal of Antituberculosis, vol. 34, no. 8, pp. 485-508, 2012.

[16] Chinese Society of Tuberculosis and Chinese Medical Association, "[Expert consensus on diagnosis and treatment of nontuberculous mycobacterium diseases]," Chinese Journal of Tuberculosis and Respiratory Diseases, vol. 35, no. 8, pp. 572-580, 2012.

[17] Y. L. Zhao and Y. Pang, [Practice for Laboratory Testing of Tuberculosis], People's Medical Publishing House, Beijing, China, 2015.

[18] J. M. Bryant, D. M. Grogono, D. Rodriguez-Rincon et al., "Emergence and spread of a human-transmissible multidrugresistant nontuberculous mycobacterium," Science, vol. 354, no. 6313, pp. 751-757, 2016.

[19] S. Simons, J. Van Ingen, P.-R. Hsueh et al., "Nontuberculous mycobacteria in respiratory tract infections, eastern Asia," Emerging Infectious Diseases, vol. 17, no. 3, pp. 343-349, 2011.

[20] S. H. Kasperbauer and M. A. De Groote, "The treatment of rapidly growing mycobacterial infections," Clinics in Chest Medicine, vol. 36, no. 1, pp. 67-78, 2015.

[21] M. M. Johnson and J. A. Odell, "Nontuberculous mycobacterial pulmonary infections," Journal of Thoracic Disease, vol. 6, no. 3, pp. 210-220, 2014.

[22] K. L. Winthrop, E. Chang, S. Yamashita, M. F. Iademarco, and P. A. LoBue, "Nontuberculous mycobacteria infections and anti-tumor necrosis factor- $\alpha$ therapy," Emerging Infectious Diseases, vol. 15, no. 10, pp. 1556-1561, 2009.

[23] P. M. Cassidy, K. Hedberg, A. Saulson, E. McNelly, and K. L. Winthrop, "Nontuberculous mycobacterial disease prevalence and risk factors: a changing epidemiology," Clinical Infectious Diseases, vol. 49, no. 12, pp. e124-e129, 2009.

[24] M. D. Yates, J. M. Grange, and C. H. Collins, "The nature of mycobacterial disease in south east England, 1977-84," Journal of Epidemiology \& Community Health, vol. 40, no. 4, pp. 295-300, 1986.

[25] Chinese Society of Tuberculosis and Chinese Medical Association, "Expert consensus on laboratory diagnosis of nontuberculous mycobacterium diseases," Chinese Journal of Tuberculosis and Respiratory Diseases, vol. 39, no. 6, pp. 438-443, 2016.

[26] Q. Y. Yang, Z. H. Xing, and Z. H. Qin, "[A comparison of CT characteristics between Mycobacterium intracellulare pulmonary disease, Mycobacterium kansasii and Mycobacterium abscessus/chelonae pulmonary disease]," Chinese Journal of Medical Imaging, vol. 35, no. 6, pp. 857-861, 2019.

[27] C. L. Daley, J. M. Iaccarino, C. Lange et al., "Treatment of nontuberculous mycobacterial pulmonary disease: an official ATS/ERS/ESCMID/IDSA clinical practice guideline," Clinical Infectious Diseases, vol. 71, no. 4, pp. e1-e36, 2020.

[28] Y. Xu, B. Liang, C. Du et al., "Rapid identification of clinically relevant mycobacterium species by multicolor melting curve analysis," Journal of Clinical Microbiology, vol. 57, no. 1, 2019.

[29] T.-S. Huang, C.-C. Lee, H.-Z. Tu, and S. S.-J. Lee, "Rapid identification of mycobacteria from positive MGIT broths of primary cultures by MALDI-TOF mass spectrometry," PLoS One, vol. 13, no. 2, Article ID e0192291, 2018.

[30] H.-Y. Kim, Y. Kook, Y.-J. Yun et al., "Proportions of $M y$ cobacterium massiliense and Mycobacterium bolletii strains 
among Korean Mycobacterium chelonae-Mycobacterium abscessus group isolates," Journal of Clinical Microbiology, vol. 46, no. 10, pp. 3384-3390, 2008.

[31] G. Li, H. Pang, Q. Guo et al., “Antimicrobial susceptibility and MIC distribution of 41 drugs against clinical isolates from China and reference strains of nontuberculous mycobacteria," International Journal of Antimicrobial Agents, vol. 49, no. 3, pp. 364-374, 2017.

[32] G. L. Woods, B. A. Brown-Elliott, P. S. Conville et al., "CLSI standards: guidelines for health care excellence, susceptibility testing of mycobacteria, nocardiae, and other aerobic actinomycetes," Clinical and Laboratory Standards Institute, Wayne, PA, USA, M24-A2, 2011.

[33] S. Obata, Z. Zwolska, E. Toyota et al., "Association of rpoB mutations with rifampicin resistance in Mycobacterium avium," International Journal of Antimicrobial Agents, vol. 27, no. 1, pp. 32-39, 2006.

[34] Y. Kobashi, K. Yoshida, N. Miyashita, Y. Niki, and M. Oka, "Relationship between clinical efficacy of treatment of pulmonary Mycobacterium avium complex disease and drugsensitivity testing of Mycobacterium avium complex isolates," Journal of Infection and Chemotherapy, vol. 12, no. 4, pp. 195-202, 2006.

[35] B. A. Brown-Elliott, E. Iakhiaeva, D. E. Griffith et al., "In vitro activity of amikacin against isolates of Mycobacterium avium complex with proposed MIC breakpoints and finding of a $16 \mathrm{~S}$ rRNA gene mutation in treated isolates," Journal of Clinical Microbiology, vol. 51, no. 10, pp. 3389-3394, 2013.

[36] M. Maiga, S. Siddiqui, S. Diallo et al., "Failure to recognize nontuberculous mycobacteria leads to misdiagnosis of chronic pulmonary tuberculosis," PLoS One, vol. 7, no. 5, Article ID e36902, 2012.

[37] S. G. Liu, X. Gao, and J. Q. Zhu, “[A retrospective analysis of nontuberculous mycobacterial pulmonary disease]," Journal of Central South University (Medical Sciences), vol. 44, no. 4, pp. 432-436, 2019.

[38] A. D. Khosravi, M. Mirsaeidi, A. Farahani et al., "Prevalence of nontuberculous mycobacteria and high efficacy of D-cycloserine and its synergistic effect with clarithromycin against Mycobacterium fortuitum and Mycobacterium abscessus," Infection and Drug Resistance, vol. 11, pp. 2521-2532, 2018.

[39] M. Dastranj, A. Farahani, A. Hashemi Shahraki, S. Atashi, and P. Mohajeri, "Molecular identification and distribution of non-tuberculous mycobacteria isolated from clinical specimens by PCR-sequencing method in west of Iran," The Clinical Respiratory Journal, vol. 12, no. 3, pp. 996-1002, 2018.

[40] F. N. Riello, R. T. S. Brígido, S. Araújo, T. A. Moreira, L. R. Goulart, and I. M. B. Goulart, "Diagnosis of mycobacterial infections based on acid-fast bacilli test and bacterial growth time and implications on treatment and disease outcome," BMC Infectious Diseases, vol. 16, no. 1, p. 142, 2016.

[41] Y. Lee, J.-W. Song, E. J. Chae et al., "CT findings of pulmonary non-tuberculous mycobacterial infection in non-AIDS immunocompromised patients: a case-controlled comparison with immunocompetent patients," British Journal of Radiology, vol. 86, no. 1024, Article ID 20120209, 2013.

[42] M.-K. Yuan, C.-Y. Chang, P.-H. Tsai, Y.-M. Lee, J.-W. Huang, and S.-C. Chang, "Comparative chest computed tomography findings of non-tuberculous mycobacterial lung diseases and pulmonary tuberculosis in patients with acid fast bacilli smear-positive sputum," BMC Pulmonary Medicine, vol. 14, no. 1, p. $65,2014$.
[43] S. G. Pereira, S. Alarico, I. Tiago et al., "Studies of antimicrobial resistance in rare mycobacteria from a nosocomial environment," BMC Microbiology, vol. 19, no. 1, p. 62, 2019.

[44] P. H. C. Candido, L. d. S. Nunes, E. A. Marques et al., "Multidrug-resistant nontuberculous mycobacteria isolated from cystic fibrosis patients," Journal of Clinical Microbiology, vol. 52, no. 8, pp. 2990-2997, 2014.

[45] S. Saxena, H. P. Spaink, and G. Forn-Cuní, "Drug resistance in nontuberculous mycobacteria: mechanisms and models," Biology, vol. 10, no. 2, 2021.

[46] S. Q. Wang, G. L. Jiang, and G. M. Wei, "[A study on drug resistance spectrum and genotype characteristics of clinically isolated strains of Mycobacterium intracellulare]," Chinese Journal of Tuberculosis and Respiratory Diseases, vol. 41, no. 7, pp. 539-543, 2018.

[47] Y. Yamaba, Y. Ito, K. Suzuki et al., "Moxifloxacin resistance and genotyping of Mycobacterium avium and Mycobacterium intracellulare isolates in Japan," Journal of Infection and Chemotherapy, vol. 25, no. 12, pp. 995-1000, 2019.

[48] E. H. Cho, H. J. Huh, D. J. Song et al., "Differences in drug susceptibility pattern between Mycobacterium avium and Mycobacterium intracellulare isolated in respiratory specimens," Journal of Infection and Chemotherapy, vol. 24, no. 4, pp. $315-318,2018$

[49] A. Cheng, Y. T. Tsai, S. Y. Chang et al., "n vitro synergism of rifabutin with clarithromycin, imipenem, and tigecycline against the Mycobacterium abscessus complex," Antimicrobial Agents and Chemotherapy, vol. 63, no. 4, 2019.

[50] M. R. Lee, W. H. Sheng, C. C. Hung, C. J. Yu, L. N. Lee, and P. R. Hsueh, "Mycobacterium abscessus complex infections in humans," Emerging Infectious Diseases, vol. 21, no. 9, pp. 1638-1646, 2015.

[51] P. Ananta, I. Kham-Ngam, P. Chetchotisakd et al., "Analysis of drug-susceptibility patterns and gene sequences associated with clarithromycin and amikacin resistance in serial $M y$ cobacterium abscessus isolates from clinical specimens from northeast Thailand," PLoS One, vol. 13, no. 11, Article ID e0208053, 2018.

[52] W. J. Nie, H. F. Duan, and H. R. Huang, "[Species identification and antimicrobial susceptibility testing of Mycobacterium abscessus]," Chinese Journal of Tuberculosis and Respiratory Diseases, vol. 37, no. 7, pp. 517-521, 2014.

[53] R. C. Lopeman, J. Harrison, M. Desai, and J. A. G. Cox, "Mycobacterium abscessus: environmental bacterium turned clinical nightmare,” Microorganisms, vol. 7, no. 3, 2019. 